\title{
Avulsão do tendão reto femoral em atleta amador: relato de caso diagnosticado pela ressonância magnética
}

\section{Rectus femoris tendon avulsion in an amateur athlete: case report diagnosed by magnetic resonance}

\author{
Thaís Amanda Frank de Almeida Alves ${ }^{1}$, Marco Antonio Alves Braun ${ }^{1}$, \\ Márcio Luís Duarte ${ }^{2}$, Lucas Ribeiro dos Santos ${ }^{3}$, \\ Marcelo de Queiroz Pereira da Silva ${ }^{4}$
}

\begin{abstract}
Resumo
Introdução: o músculo reto femoral é o músculo mais frequentemente lesado do grupo quadríceps durante chutes e corridas repetitivas, apesar de suas avulsões serem raras. A dor localizada na coxa proximal e a incapacidade de flexionar o quadril e/ou estender o joelho associado à história do paciente de contração violenta ou alongamento forçado do grupo muscular do quadríceps femoral podem indicar uma avulsão do reto femoral. Objetivo: relatar um caso e revisar a literatura acerca desta lesão incomum. Materiais e Métodos: revisão do prontuário, registro fotográfico do método diagnóstico e revisão da literatura. Resultados: homem de 46 anos com dor localizada no quadril esquerdo com irradiação para a coxa por três meses após cair no chão com a região dolorida em um jogo de futebol. A dor começou subitamente uma semana após a queda. A ressonância magnética do quadril apresentava avulsão completa do reto femoral esquerdo, distando $2 \mathrm{~cm}$ da espinha ilíaca anterior inferior com efusão líquida adjacente. Conclusão: este relato demonstra a dificuldade de diagnosticar essa lesão, pois o seu exame físico é inespecífico, podendo simular patologias mais complexas, necessitando de exames complementares para seu correto diagnóstico.
\end{abstract}

Palavras-chave: Traumatismos dos tendões; Imagem de ressonância magnética; Músculo quadríceps; Coxa da perna.

\begin{abstract}
Introduction: the rectus femoris muscle is the most frequently injured muscle of the quadriceps group during repetitive kicking and sprinting. Avulsions of the rectus femoris are rare injuries. Pain located at the proximal thigh and disability in flexing the hip and/or extending the knee associated with a patient history of a violent contraction or forceful stretching of the quadriceps femoris muscle
\end{abstract}

${ }^{1}$ Graduandos em Medicina no Centro Universitário Lusíada (UNILUS), Santos, São Paulo, Brasil.

${ }^{2}$ Médico Especialista em Radiologia e Diagnóstico por Imagem pelo Colégio Brasileiro de Radiologia e Diagnóstico por Imagem (CBR), São Paulo, São Paulo, Brasil. Mestrado em Saúde Baseada em Evidências pela Universidade Federal de São Paulo (Unifesp), São Paulo, São Paulo, Brasil. Médico na Webimagem Telerradiologia, São Paulo, São Paulo, Brasil. E-mail: marcioluisduarte@gmail.com

${ }^{3}$ Médico Endocrinologista. Professor de Fisiologia e Medicina Interna do Centro Universitário Lusíada, Santos, São Paulo, Brasil. Mestrado em Saúde Baseada em Evidências pela Universidade Federal de São Paulo, São Paulo, São Paulo, Brasil.

${ }^{4}$ Médico Especialista em Radiologia e Diagnóstico por Imagem pelo Colégio Brasileiro de Radiologia e Diagnóstico por Imagem, São Paulo, São Paulo, Brasil. Médico na Webimagem Telerradiologia, São Paulo, São Paulo, Brasil. 
group can indicate an avulsion of rectus femoris. Objective: report a case and review the literature about this uncommon lesion. Materials and Methods: we reviewed medical records, photographic records of diagnostic methods, and reviews from the literature. Results: a 46-year-old man with pain located at the left anterior hip with irradiation to the thigh for three months after falling to the ground with the sore region in a soccer game. The pain started suddenly one week after the fall. The MRI of the hip featured complete avulsion of the left rectus femoris, being $2 \mathrm{~cm}$ distal from the anterior inferior iliac spine with adjacent liquid effusion. Conclusion: this report demonstrates the difficulty of diagnosing this lesion, since its physical examination is non-specific, and it can simulate more complex pathologies, requiring complementary tests for its correct diagnosis.

Keywords: Tendon injuries; Magnetic resonance imaging; Quadriceps muscle; Thigh.

\section{Introdução}

O músculo reto femoral é o músculo mais frequentemente lesado do quadríceps durante chutes e corridas repetitivas. ${ }^{(1)} \mathrm{O}$ tendão da cabeça direta é superficial e se origina da espinha ilíaca anteroinferior e o tendão da cabeça indireta é profundo e se origina da margem superior acetabular. ${ }^{(2)}$ As principais ações do músculo reto femoral são a extensão do joelho e a flexão do quadril. ${ }^{(3)}$ As características do cruzamento de duas articulações e uma alta porcentagem de fibras musculares tipo II são as principais responsáveis pela desaceleração; assim, quando esses agem acentuadamente são comumente associados a músculos lesionados. ${ }^{(2)} \mathrm{O}$ padrão das lesões do reto femoral varia de pequenas roturas a roturas completas de sua porção tendínea ou avulsões em sua origem. ${ }^{(4-8)}$ As avulsões proximais do reto femoral são lesões raras. ${ }^{(8-11)}$

A dor localizada na coxa proximal e incapacidade de flexionar o quadril e/ou estender o joelho associado à história do paciente de contração violenta ou alongamento forçado do grupo muscular do quadríceps femoral podem indicar uma avulsão da cabeça direta do reto femoral. ${ }^{(3)}$ Também pode ser observada no exame físico discreta massa na porção anterior da coxa relacionada à retração muscular, podendo ser confundida com uma neoplasia de partes moles. ${ }^{(12)}$

A ressonância magnética ( $\mathrm{RM})$ é o padrãoouro para diagnosticar patologias do quadril com dor na virilha. ${ }^{(13)}$ Exames de imagem como a RM e a ultrassonografia são importantes para acompanhar o processo de cicatrização. ${ }^{(13)}$ Este relato de caso é sobre e avulsão da cabeça direta do reto em um paciente atleta amador de 46 anos.

\section{Relato do caso}

Homem de 46 anos refere dor súbita na região anterior do quadril esquerdo com irradiação para a coxa há três meses. Informa que a dor piora quando fica muito tempo sentado. Refere queda ao chão com a região dolorida em jogo de futebol, porém informa que a dor na região começou apenas uma semana depois do referido trauma. Nega doenças prévias e limitação funcional. Pratica futebol uma vez por semana e caminhada de $6 \mathrm{~km}$ três vezes por semana. Não apresenta alteração da coloração da pele e edema durante o exame físico, porém apresenta dor na região anterior do quadril com a hiperextensão do quadril e flexão do joelho - teste de Ely positivo. Apresenta duas cirurgias anteriores - ombro direito há cinco anos e tornozelo esquerdo há oito anos.

A RM apresenta avulsão completa da cabeça direta do tendão reto femoral esquerdo, com efusão líquida adjacente, distando cerca de $2 \mathrm{~cm}$ da espinha ilíaca anteroinferior (Figura 1). Com o diagnóstico, pelo exame de imagem o paciente foi encaminhado para o ortopedista para tratamento cirúrgico. 
Figura 1 - Ressonância magnética na sequência T2 com saturação de gordura no corte coronal em A, axial em B e sagital em $\mathrm{C}$ demonstrando avulsão completa da cabeça direta do tendão reto femoral esquerdo com efusão líquida local (círculo branco).

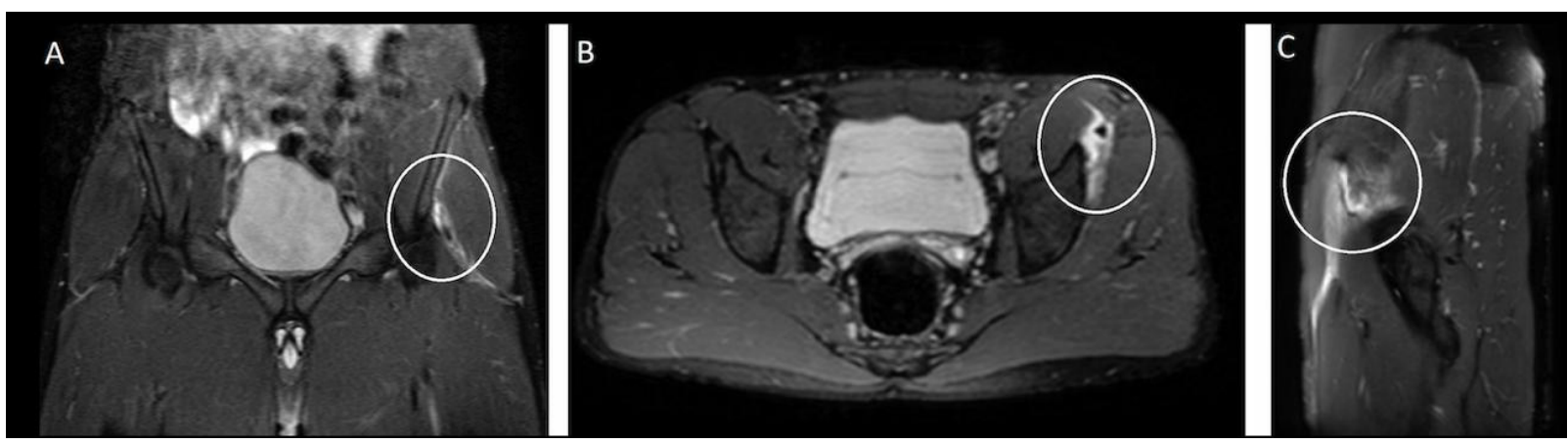

Fonte: os autores.

\section{Discussão}

O músculo reto femoral é o componente mais frequentemente lesado do grupo muscular do quadríceps e possui muitas das características dos músculos lesionados. ${ }^{(2)} \mathrm{A}$ avulsão proximal do tendão do músculo reto femoral é uma lesão rara. ${ }^{(3)}$ Os fatores predisponentes incluem fadiga muscular, exercícios de warm-up insuficientes, mau condicionamento muscular geral e rotura prévia. ${ }^{(14)}$ Lesões no reto femoral são raras na população adulta e ocorrem mais comumente em atletas. ${ }^{(15)}$ Por exemplo, as lesões por avulsão no reto representam $1,5 \%$ das lesões no quadril em atletas da National Football League (NFL) entre 1997 e 2006. ${ }^{(15)}$

Os mecanismos relatados, principalmente, para as lesões são contrações musculares violentas ou explosivas contra resistência fixa e desaceleração súbita ou alongamento muscular como resultado de um trauma direto. ${ }^{(16-17)}$ Isso geralmente ocorre durante movimentos como chutar uma bola ou correr. ${ }^{(2,18-21)}$ A causa mais comum de dor aguda na virilha em atletas é uma lesão musculoesquelética, como uma tensão muscular. ${ }^{(16)}$ No entanto, o diagnóstico diferencial pode incluir uma variedade de causas. ${ }^{(16)}$

Pacientes com lesões agudas da junção músculotendinosa profunda apresentam histórico de chutes ou corridas resultantes em dor localizada na coxa proximal e incapacidade de flexionar o quadril e/ou estender o joelho. ${ }^{(13)}$ Dor persistente, sensibilidade e assimetria do reto femoral podem estar presentes no exame físico. ${ }^{(22-25)}$ Uma massa anterior discreta na coxa relacionada à retração muscular pode ser confundida com uma neoplasia de partes moles. ${ }^{(22-25)}$

A RM é o exame padrão-ouro para diagnosticar patologias do quadril com dor na virilha. ${ }^{(13)} \mathrm{Os}$ exames de imagem, como a RM e a ultrassonografia, são importantes para acompanhar o processo de cicatrização, detectar a ocorrência de complicações e reduzir a duração da inatividade esportiva. ${ }^{(13)} \mathrm{O}$ padrão das lesões do reto femoral varia de pequenas roturas a roturas completas de sua porção tendinosa ou avulsões em sua origem. ${ }^{(1,5-6,8,10)}$ Um padrão comum de rotura da RM é o sinal do bull's-eye alto sinal ao redor do tendão observado em múltiplas imagens axiais consecutivas nas sequências sensíveis ao líquido. ${ }^{(22)}$ Esse alto sinal pode estar presente em casos agudos e crônicos. ${ }^{(22)}$

O sinal do bull's-eye sugere estágios de lesão e cicatrização em torno do tendão. ${ }^{(12)}$ Inicialmente, representa edema e hemorragia. ${ }^{(12)}$ Posteriormente, e após a injeção intravenosa de gadolínio, o alto sinal pode refletir aumento da vascularização e cicatrização. ${ }^{(12)} \mathrm{O}$ sinal do bull's-eye com atrofia secundária e a infiltração gordurosa do músculo 
ao redor do tendão reflete lesão remota. ${ }^{(12)}$ Outros achados da RM incluem retrações, hematoma, pseudocisto, reposição muscular focal gordurosa e cicatrizes longitudinais. ${ }^{(12)}$

Não há protocolo definitivo de tratamento para avulsão da cabeça direta do reto femoral. ${ }^{(16)}$ Dean et al. indicaram tratamento cirúrgico para atletas de alto nível e na falha no tratamento não operatório (dor e fraqueza por mais de três meses), enquanto contraindicado em avulsões não deslocadas, roturas sem retração e pacientes de baixa demanda nos quais as roturas crônicas não interferem na função diária normal. ${ }^{(26-27)}$ Essas indicações também estão alinhadas com nosso relato de caso.

\section{Referências}

1 Ueblacker P, Müller-Wohlfahrt HW, Hinterwimmer $\mathrm{S}$, Imhoff AB, Feucht MJ. Suture anchor repair of proximal rectus femoris avulsions in elite football players. Knee Surg Sports Traumatol Arthrosc. 2015 Sep;23(9):2590-4. doi: 10.1007/ s00167-014-3177-0.

2 Hasselmann CT, Best TM. An explanation for various rectus femoris strain injuries using previously undescribed muscle architecture. Am J Sports Med. 1995;23(4):493-9.

3 Irmola T, Heikkilä JT, Orava S, Sarimo J. Total proximal tendon avulsion of the rectus femoris muscle. Scand J Med Sci Sports. 2007 Aug;17(4):378-82. doi: 10.1111/j.1600-0838. 2006.00564.x.

4 Garcia VV, Duhrkop DC, Seijas R, Ares O, Cugat R. Surgical treatment of proximal ruptures of the rectus femoris in professional soccer players. Arch Orthop Trauma Surg. 2012 Mar;132(3):329-33. doi: 10.1007/s00402-011$1372-8$.

5 Hughes C, Hasselman CT, Best TM, Martinez $\mathrm{S}$, Garrett WE Jr. Incomplete, intrasubstance strain injuries of the rectus femoris muscle. Am J Sports Med. 1995;23:500-6.
6 Irmola T, Heikkila JT, Orava S, Sarimo J. Total proximal tendon avulsion of the rectus femoris muscle. Scand J Med Sci Sports. 2007; 17(4):378-82.

7 Mendiguchia J, Alentorn-Geli E, Idoate F, Myer GD. Rectus femoris muscle injuries in football: a clinically relevant review of mechanisms of injury, risk factors and preventive strategies. Br J Sports Med. 2013;47(6):359-66.

8 Ouellette H, Thomas BJ, Nelson E, Torriani M. MR imaging of rectus femoris origin injuries. Skeletal Radiol. 2006;35(9):665-72.

9 Gamradt SC, Brophy RH, Barnes R, Warren RF, Thomas Byrd JW, Kelly BT. Nonoperative treatment for proximal avulsion of the rectus femoris in professional American football. Am J Sports Med. 2009;37(7):1370-74.

10 Garcia VV, Duhrkop DC, Seijas R, Ares O, Cugat R. Surgical treatment of proximal ruptures of the rectus femoris in professional soccer players. Arch Orthop Trauma Surg. 2012; 132(3):329-33

11 Hsu JC, Fischer DA, Wright RW. Proximal rectus femoris avulsions in national football league kickers: a report of 2 cases. Am J Sports Med. 2005;33(7):1085-7

12 Gyftopoulos S, Rosenberg ZS, Schweitzer ME, Bordalo-Rodrigues M. Normal Anatomy and Strains of the Deep Musculotendinous Junction of the Proximal Rectus Femoris: MRI Features. AJR Am J Roentgenol. 2008 Mar;190(3):W182-6. doi: 10.2214/AJR.07. 2947.

13 Ouellette H, Thomas BJ, Nelson E, Torriani M. MR Imaging of Rectus Femoris Origin Injuries. Skeletal Radiol. 2006 Sep;35(9):665-72. doi: 10.1007/s00256-006-0162-9.

14 Garrett WE Jr. Muscle strain injuries. Am J Sports Med. 1996;24[suppl 6]:S2-S8.

15 Hapa O, Bedi A, Gursan O, et al. Anatomic footprint of the direct head of the rectus femoris origin: cadaveric study and clinical series of hips after arthroscopic anterior inferior iliac spine/subspine decompression. Arthroscopy. 2013;29:1932-40. 
16 Lasse L, Jussi K, Ricard P, Jordi P, Juha R, Sakari O. Operative Treatment of Proximal Rectus Femoris Injuries in Professional Soccer Players: A Series of 19 Cases. Orthop J Sports Med. 2018;Oct;6(10).

17 Yildiz C, Aydin T, Yildiz Y, Kalyon TA, Basbozkurt M. Anterior inferior iliac spine apophyseal avulsion fracture. J South Orthop Assoc. 2003;12(1):38-40.

18 Orchard JW. Intrinsic and extrinsic risk factors for muscle strains in australian football. Am J Sports Med. 2001;29(3):292-7.

19 Brophy RH, Wright RW, Powell JW, Matava MJ. Injuries to kickers in American football: the National Football League experience. Am J Sports Med. 2010;38(6):1166-73.

20 Ekstrand J, Hagglund M, Walden M. Epidemiology of muscle injuries in professional football (soccer). Am J Sports Med. 2011; Jun;39(6):1226-32. doi: 10.1177/0363546510 395879 .

21 Ekstrand J, Hagglund M, Walden M. Injury incidence and injury patterns in professional football: the UEFA injury study. Br J Sports Med. 2011;45(7):553-8.

22 Hughes CT, Hasselman CT, Best TM, Martinez S, Garrett WE Jr. Incomplete, intrasubstance strain injuries of the rectus femoris muscle. Am J Sports Med. 1995;Jul-Aug;23(4):500-6. doi: $10.1177 / 036354659502300422$.

23 Bordalo-Rodrigues M, Rosenberg ZS. MR imaging of the proximal rectus femoris musculotendinous unit. Magn Reson Imaging Clin N Am. 2005;13:717-25.

24 Hughes CT, Hasselman CT, Best TM, Martinez $\mathrm{S}$, Garrett WE Jr. An explanation for various rectus femoris strain injuries using previously undescribed muscle architecture. Am J Sports Med. 1995;23:493-9.

25 Temple HT, Kuklo TR, Sweet DE, Gibbons CL, Murpheyet MD. Rectus femoris muscle tear appearing as a pseudotumor. Am J Sports Med. 1998;Jul-Aug;26(4):544-8. doi: 10.1177/ 03635465980260041301 .
26 Lempainen L, Kosola J, Pruna R, Puigdellivol $\mathrm{J}$, Ranne J, Orava S. Operative treatment of proximal rectus femoris injuries in professional soccer players: a series of 19 cases. Orthop J Sports Med. 2018 Oct; 2;6(10):2325967118 798827.

27 Dean CS, Arbeloa-Gutierrez L, Chahla J, Pascual-Garrido C. Proximal rectus femoris avulsion repair. Arthrosc Tech. 2016;5:e545-9. 
Alves, T. A. F. A. et al. 